\title{
Special issue on dynamic textures in video
}

\author{
A. Enis Cetin • Fatih Porikli
}

Published online: 17 February 2011

(C) Springer-Verlag 2011

Two-dimensional textures in images have been extensively studied in the past. On the other hand, there is comparatively limited research on three-dimensional dynamic textures that exhibit certain time-varying properties in video. In many scenes there are regions having significant structural similarity and exhibit high temporal correlations between image frames forming the video [1]. A tree swaying in the wind or a wave lapping on a beach is not just a collection of randomly shuffled appearances, but a physical system that has characteristic responses associated with its dynamics. Examples of such dynamic phenomena include flames, smoke, sea, waves, clouds, fog, crowds in public places and sports events, some human movements, and even shadows [1-9]. It is known that dynamic textures, especially for outdoor scenes, cause major problems in motion detection and analysis tasks. Besides, they drastically decrease the coding efficiency of video encoders although they do not contain any useful and discriminative information. They complicate motion-based object recognition methods. By segmenting and excluding dynamic textures, the robustness of the moving object detection and action identification can be improved. Other practical applications include detection of certain types of dynamic textures such as fire and smoke, realistic rendering and compact visualization of dynamic textures, and efficient retrieval of video in multimedia databases. The

\footnotetext{
A. Enis Cetin $(\varangle)$

Department of Electrical and Electronics Engineering, Bilkent University, Ankara, Turkey

e-mail: cetin@bilkent.edu.tr

F. Porikli

Mitsubishi Electric Research Labs, MERL, Cambridge, USA

e-mail: fatih@merl.com
}

objective of this special issue is to provide a comprehensive overview of theoretical and practical aspects as well as collate and disseminate the state of the art research results on dynamic textures.

This special issue consists of five papers. Articles by Chetverikov et al. [4] and Chan et al. [5] address two related problems about foreground and background segmentation in video using dynamic texture models. Peteri describes a particle filtering-based dynamic texture tracking method in video [6]. Kellokumpu et al. [7] presents a human action recognition method using dynamic texture descriptors. In [8], Dixon et al. present an application of texture methods in industrial machine vision. Another related article which recently appeared in this journal is about flame detection [9].

\section{References}

1. Chetverikov, D., Peteri, R.: A brief survey of dynamic texture description and recognition. Comput. Recognit. Syst. Adv. Soft Comput. 30, 17-26 (2005). doi:10.1007/3-540-32390-2_2

2. Phillips, W. III., Shah, M., da Lobo, N.V.: Flame recognition in video. Pattern Recognit. Lett. 23(1-3), 319-327 (2002)

3. Töreyin, B.U., Dedeoglu, Y., Güdükbay, U., Çetin Enis, A.: Computer vision based method for real-time fire and flame detection. Pattern Recognit. Lett. 27(1), 49-58 (2006)

4. Chetverikov, D., Fazekas S., Haindl, M.: Dynamic texture as foreground and background. Mach. Vis. Appl. doi:10.1007/ s00138-010-0251-6

5. Chan, A.B., Mahadevan, V., Vasconcelos, N.: Generalized StaufferGrimson background subtraction for dynamic scenes. Mach. Vis. Appl. doi:10.1007/s00138-010-0262-3

6. Péteri, R.: Tracking dynamic textures using a particle filter driven by intrinsic motion information. Mach. Vis. Appl. doi:10.1007/ s00138-009-0236-5

7. Kellokumpu, V., Zhao, G., Pietikäinen, M.: Recognition of human actions using texture descriptors. Mach. Vis. Appl. doi:10.1007/ s00138-009-0233-8 
8. Dixon, M., Glaubius, R., Freeman, P., Pless, R., Gleason, M.P., et al.: Measuring optical distortion in aircraft transparencies: a fully automated system for quantitative evaluation. Mach. Vis. Appl. doi:10. 1007/s00138-010-0258-z
9. Yuan, F.N.: An integrated fire detection and suppression system based on widely available video surveillance. Mach. Vis. Appl. 21(6), 941-948 (2010) 